\title{
Competing contact processes in the Watts-Strogatz network
}

\author{
Marcin Rybak, Krzysztof Malarz ${ }^{\mathrm{a}}$, and Krzysztof Kułakowski \\ AGH University of Science and Technology, Faculty of Physics and Applied Computer Science, al. Mickiewicza 30, \\ 30-059 Krakow, Poland
}

Received 4 March 2016 / Received in final form 15 April 2016

Published online 8 June 2016

(c) The Author(s) 2016. This article is published with open access at Springerlink.com

\begin{abstract}
We investigate two competing contact processes on a set of Watts-Strogatz networks with the clustering coefficient tuned by rewiring. The base for network construction is one-dimensional chain of $N$ sites, where each site $i$ is directly linked to nodes labelled as $i \pm 1$ and $i \pm 2$. So initially, each node has the same degree $k_{i}=4$. The periodic boundary conditions are assumed as well. For each node $i$ the links to sites $i+1$ and $i+2$ are rewired to two randomly selected nodes so far not-connected to node $i$. An increase of the rewiring probability $q$ influences the nodes degree distribution and the network clusterization coefficient $\mathcal{C}$. For given values of rewiring probability $q$ the set $\mathcal{N}(q)=\left\{\mathcal{N}_{1}, \mathcal{N}_{2}, \ldots, \mathcal{N}_{M}\right\}$ of $M$ networks is generated. The network's nodes are decorated with spin-like variables $s_{i} \in\{S, D\}$. During simulation each $S$ node having a $D$-site in its neighbourhood converts this neighbour from $D$ to $S$ state. Conversely, a node in $D$ state having at least one neighbour also in state $D$-state converts all nearest-neighbours of this pair into $D$-state. The latter is realized with probability $p$. We plot the dependence of the nodes $S$ final density $n_{S}^{T}$ on initial nodes $S$ fraction $n_{S}^{0}$. Then, we construct the surface of the unstable fixed points in $\left(\mathcal{C}, p, n_{S}^{0}\right)$ space. The system evolves more often toward $n_{S}^{T}=1$ for $\left(\mathcal{C}, p, n_{S}^{0}\right)$ points situated above this surface while starting simulation with $\left(\mathcal{C}, p, n_{S}^{0}\right)$ parameters situated below this surface leads system to $n_{S}^{T}=0$. The points on this surface correspond to such value of initial fraction $n_{S}^{*}$ of $S$ nodes (for fixed values $\mathcal{C}$ and $p$ ) for which their final density is $n_{S}^{T}=\frac{1}{2}$.
\end{abstract}

\section{Introduction}

In computational modeling, the contact processes $(\mathrm{CPs})$ are dynamic systems on discrete media, where the time evolution of a local state towards survival or extinction of particles is determined by the state of the direct neighborhood of a lattice cell or of a network node. A simple realization is the voter model [1], where a particle creates another particle in its direct neighborhood. CPs have been introduced in 1974 as a toy model of spread of epidemic on a lattice [2,3]. Since then, they evolved to a frame for models in different areas, from symbiotic interactions [4] to population [5] or opinion dynamics [6]. Yet, their important role is also to inspire theoretical considerations on non-equilibrium processes [7-9]. In both these roles, the spectrum of particular realizations of CPs has been remarkably enriched. In particular, the pair contact processes have been proposed in reference [10]; there, a pair of particles annihilate or create a neighbor particle.

The aim of this paper is to report our numerical results on competing CPs of two different kinds. As far as we know, this case has not been analyzed, with two our texts [11-13] as an exception. In literature, applications

\footnotetext{
${ }^{a}$ e-mail: malarz@agh.edu.pl
}

of competing CPs are of recent interest [14-16]; yet, in all these approaches the competing processes are of the same kind. In references [11,12], the competition has been investigated between the voter model and the pair contact process without annihilation. The role of network topology has been analyzed by a comparison of results for the Watts-Strogatz network [17] and the Erdős-Rényi network [18], where the clustering coefficient $\mathcal{C}$ has been tuned in both networks. Our motivation in references $[11,12]$ was to evaluate the efficiency of the pair contact process by balancing it with the voter model of controlled efficiency; the control was kept by tuning the probability $p$ of the one-node voter dynamics. The key result of references $[11,12]$ was a phase diagram on the plane $(\mathcal{C}, p)$; below some critical line $p_{1}^{c}(\mathcal{C})$, the pair process dominates, while above another critical line $p_{2}^{c}(\mathcal{C})$, the voter dynamics prevails. Between these lines, i.e. for $p_{1}^{c}(\mathcal{C})<p<p_{2}^{c}(\mathcal{C})$, the time of relaxation was too long to get a definitive conclusion on the stability of this or that phase. Both critical lines have been found to depend on the network topology.

In paper [13], preliminary results have been reported on the competition between the invasion process $[19,20]$ dynamics and the Sznajd model dynamics $[21,22]$. The latter algorithm bears some resemblance to the pair CP [10]; 


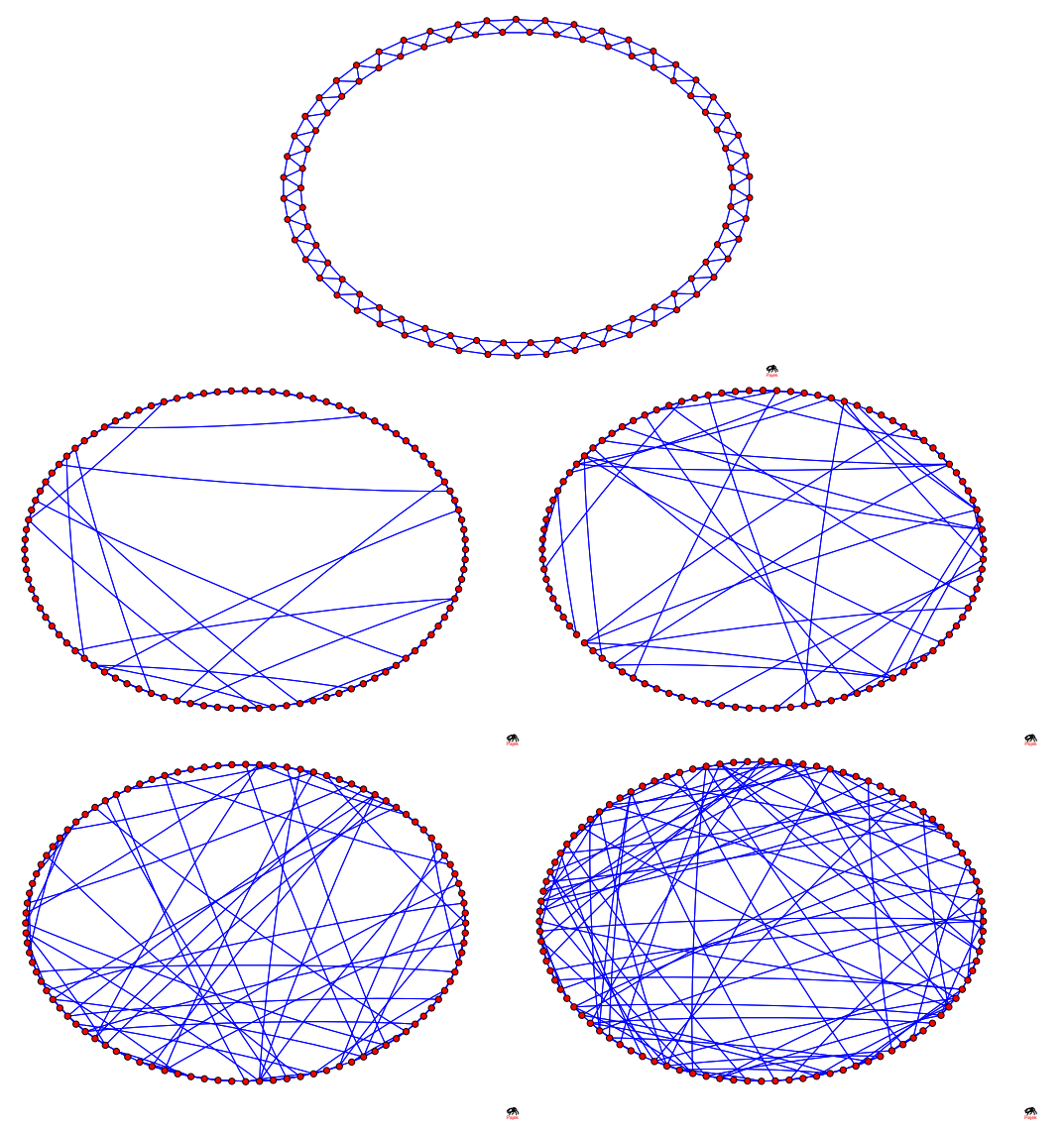

Fig. 1. Sketch of network topology for various clustering coefficients $C_{i}$. Subsequent subfigures correspond to $C_{i}=$ 0.5, 0.4, 0.3, 0.2, 0.1 in typewriter order. The figures were generated with Pajek software [27].

yet, pairs do not annihilate there, and new particles are created at the whole neighborhood of the pair. As the result, we have got a slight dependence of the transition line in the phase diagram $(\mathcal{C}, p)$ on the initial conditions, i.e. on the initial percentage of nodes in the state activated by the invasion process. As we demonstrate below, this result appears to be generic. The work presented here is entirely devoted to the role of the initial conditions. This makes the problem more complex; the plane $(C, p)$ to construct the phase diagram is to be substituted by the three-dimensional space $\left(\mathcal{C}, p, n_{S}^{0}\right)$.

The next Section 2 is devoted to the model and to the details of our simulation procedure. Section 3 provides our numerical results. In the last Section 4 we give a summary, supplemented by a note on a possible application of the scheme presented here.

\section{Model}

\subsection{Network construction}

The simulations take place on networks similar to WattsStrogatz networks [17]. The base for network construction is one-dimensional chain of $N$ sites, where each site $i$ is directly linked to nodes labeled as $i \pm 1$ and $i \pm 2$.
So initially, each node has the same degree $k_{i}=4$. The periodic boundary conditions are assumed as well.

For each node $i$ the links to sites $i+1$ and $i+2$ are rewired to two randomly selected nodes so far notconnected to node $i$. The rewiring procedure occurs with probability $q$. The examples of original and rewired network are presented in Figure 1. Increasing rewiring probability $q$ influence the nodes degree distribution and the network clusterization coefficient. Please note however, that rewiring procedure does not change average nodes degree, i.e. $\langle k\rangle=N^{-1} \sum_{i=1}^{N} k_{i}=4$.

For given values of rewiring probability $q$ the set $\mathcal{N}(q)=\left\{\mathcal{N}_{1}, \mathcal{N}_{2}, \ldots, \mathcal{N}_{M}\right\}$ of $M$ networks is generated. The clusterization coefficient $C_{j}$ for $j$ th network is defined as the average over nodes $i=1, \ldots, N$ of the local coefficient $c_{i}$, where

$$
c_{i}=\frac{2 y_{i}}{k_{i}\left(k_{i}-1\right)},
$$

and $k_{i}$ is the degree of $i$ th node, i.e. the number of nodes linked to $i$, and $y_{i}$ is the actual number of links between these $k_{i}$ nodes [17]. The clusterization coefficients $C_{j}$ for each networks in set $\mathcal{N}(q)$ do not differ more than $\delta C=$ 0.01 from average values $\mathcal{C}(q)=M^{-1} \sum_{j=1}^{M} C_{j}$.

The clusterization coefficient for unrewired $(q=0)$ network is exactly equal to $C_{j}(q=0)=\frac{1}{2}$. 


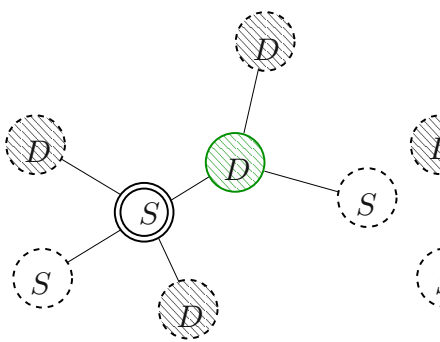

(a)

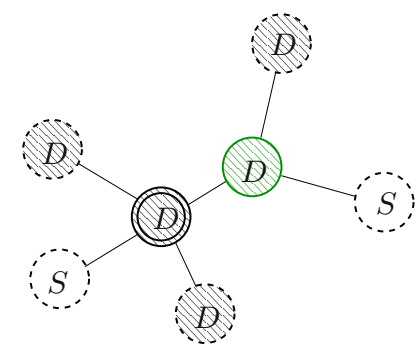

(c)

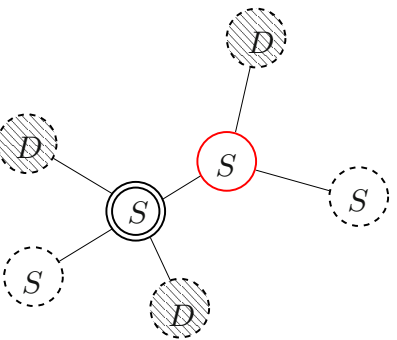

(b)

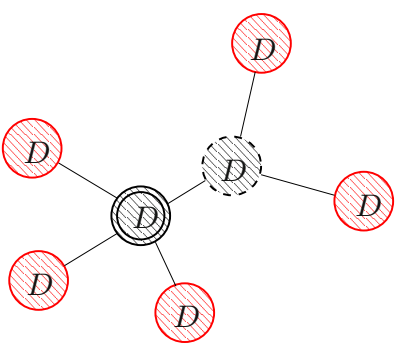

(d)
Fig. 2. Sketch of contact rules. (a) The site $S$ influences its nearest-neighbor being in state $D$. (b) As a result the neighbor is converted to $S$ state. A pair of connected nodes in $D$ state (c) influence all pair's neighbors which are changed to $D(\mathrm{~d})$. The latter occurs with probability $p$.

\subsection{Contact process description}

The network's nodes are decorated with spin-like variable $s_{i} \in\{S, D\}$. Initially (for $t=0$ ) the $S$ value is randomly assigned to the fraction of $n_{S}^{0} \equiv n_{S}(t=0)$ nodes. The remaining $\left(1-n_{S}^{0}\right) N$ nodes are assumed to be in $D$ state.

Every time step $(1 \leq t \leq T)$ the random sequence of $N$ nodes' labels is created by sampling with replacement. Now, network vertices are visited accordingly to this list.

If the visited node (denoted with double ring in Fig. 2) is marked

- as $S$ and at least one of its neighbors is in $D$ state

(Fig. 2a) then the state of this $D$ node is changed to $S$ (Fig. 2b);

- as $D$ and at least one of its neighbors is in $D$ state

(Fig. 2c) then with the probability $p$ the state of all the nearest-neighbors of this pair is changed to $D$ (Fig. 2d).

The simulation time $T$ should be long enough to ensure reaching stationary state, i.e. $d n_{S}(t \rightarrow T) / d t=0$.

\section{Results}

In Figure 3 the time evolution of fraction of $S$ nodes $n_{S}(t)$ are presented. For a given set of $\left(\mathcal{C}, p, n_{S}^{0}\right)$ parameters the results of simulation of the contact process described in Section 2.2 are averaged over $M=10^{3}$ networks realizations. These networks differ both in their topology and initial distribution of $S$ nodes. Please note however, that for

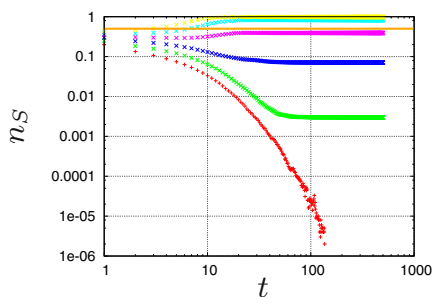

(a) $\mathcal{C}=0.1, p=0.3$

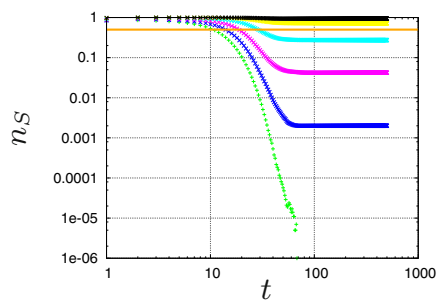

(c) $\mathcal{C}=0.4, p=0.6$

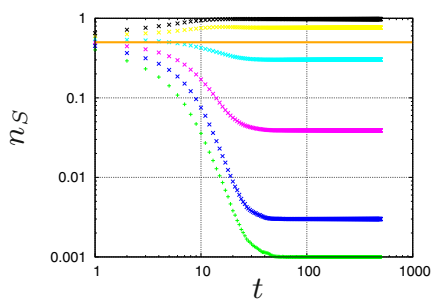

(b) $\mathcal{C}=0.2, p=0.4$

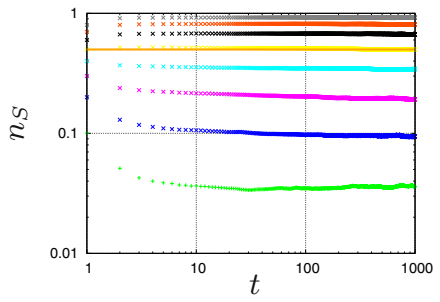

(d) $\mathcal{C}=0.5, p=0.41$
Fig. 3. Examples of the time evolution of nodes $S$ density $n_{S}(t)$ for various initial concentrations $n_{S}^{0}$ and several set of $(\mathcal{C}, p)$. The sub-figures correspond to $(\mathcal{C}, p)$ pairs equal to $(0.1,0.3),(0.2,0.4),(0.4,0.6)$ and $(0.5,0.41)$. The orange horizontal line indicates $n_{S}=\frac{1}{2}$.

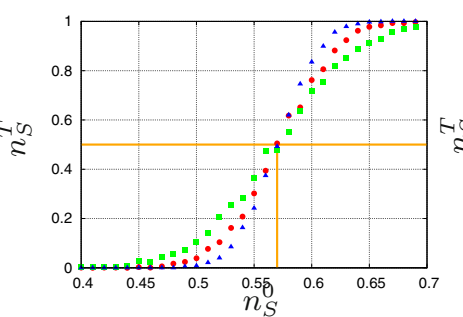

(a) $\mathcal{C}=0.2, p=0.4$

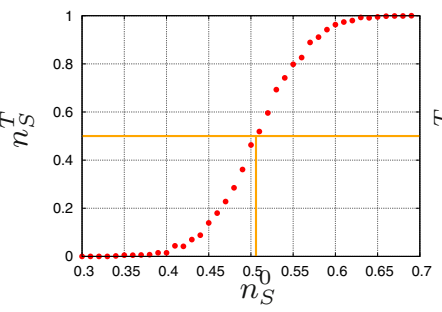

(c) $\mathcal{C}=0.4, p=0.4$

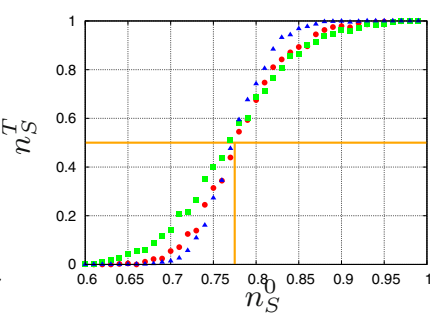

(b) $\mathcal{C}=0.3, p=0.5$

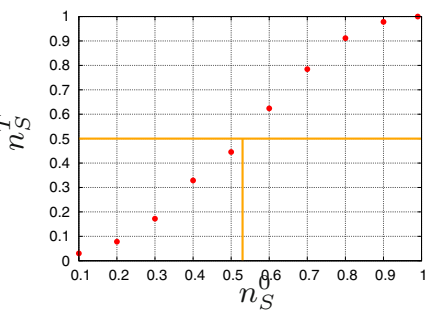

(d) $\mathcal{C}=0.5, p=0.41$
Fig. 4. Examples of the dependence of the nodes $S$ final density $n_{S}^{T}$ on initial nodes $S$ fraction $n_{S}^{0}$. The curves correspond to various network sizes $N=500(\mathbf{\square}), 1000(\mathbf{\bullet}), 2000(\mathbf{\Delta})$. The sub-figures correspond to $(\mathcal{C}, p)$ pairs equal to $(0.2,0.4)$, $(0.3,0.5),(0.4,0.4)$ and $(0.5,0.41)$.

unrewired network $(q=0, \mathcal{C}=1 / 2)$ only initial distribution of $S$ nodes allows for distinguishing among networks.

In Figure 4 the dependence of the nodes $S$ final density $n_{S}^{T} \equiv n_{S}(t=T)$ on initial nodes $S$ fraction $n_{S}^{0}$ are presented. The curves in Figure $4 \mathrm{a}$ and $4 \mathrm{~b}$ correspond to various network sizes $N=500,1000,2000$. With enlarging the system size $N$ we expect that these curves become 


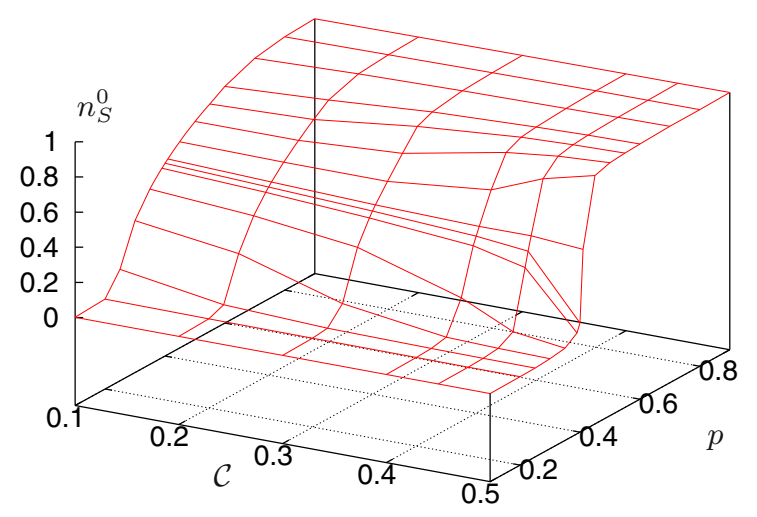

Fig. 5. The surface of the unstable fixed points in $\left(\mathcal{C}, p, n_{S}^{0}\right)$ space.

more steeper and steeper tending to Heaviside's function

$$
n_{s}^{T}\left(n_{S}^{0}\right) \asymp H\left(n_{S}^{0}-n_{S}^{*}\right)
$$

in thermodynamical limit, i.e. for $N \rightarrow \infty$. The common cross point for these curves $n_{S}^{*}$ indicate the (unstable) fixed point splitting $n_{S}^{0}$ parameter space into two regions:

- for $n_{S}^{0}<n_{S}^{*}$ the system evolves more often towards a final state with all nodes in $D$-state $\left[n_{S}^{T}=0\right]$;

- while for $n_{S}^{0}>n_{S}^{*}$ the systems prefers reaching final state with all nodes in $S$-state $\left[n_{S}^{T}=1\right]$.

Please note, that ordinates of these points are equal to $n_{S}^{T}\left(n_{S}^{*}\right) \approx \frac{1}{2}$. This yields a convenient way for rough estimation of abscissas of fixed point basing only on $n_{S}^{T}$ vs. $n_{S}^{0}$ dependence for single network size (here $N=10^{3}$ ).

The surface of the unstable fixed points in $\left(\mathcal{C}, p, n_{S}^{0}\right)$ space is presented in Figure 5 . The system evolves more likely towards $n_{S}^{T}=1$ for $\left(\mathcal{C}, p, n_{S}^{0}\right)$ points situated above this surface while points below this surface lead the system more often to $n_{S}^{T}=0$. The points on this surface correspond to such value of initial fraction $n_{S}^{*}$ of $S$ nodes (for fixed values $\mathcal{C}$ and $p$ ) for which their final density is $n_{S}^{T}=\frac{1}{2}$. Of course, reaching the final concentration $n_{S}^{T}$ of $S$ nodes exactly equal to $\frac{1}{2}$ is rather rare. Thus, we estimate $n_{S}^{*}$ as

$$
n_{S}^{*} \approx \frac{n_{S}^{-}(0)\left[\frac{1}{2}-n_{S}^{+}(T)\right]+n_{S}^{+}(0)\left[n_{S}^{-}(T)-\frac{1}{2}\right]}{n_{S}^{-}(T)-n_{S}^{+}(T)},
$$

where $n_{S}^{ \pm}(T)$ are the values of $n_{S}^{T}$ closest to $\frac{1}{2}$ and obeying inequality $n_{S}^{-}(T)<\frac{1}{2}<n_{S}^{+}(T)$ while $n_{S}^{ \pm}(0)$ are corresponding initial concentration of $S$ nodes leading to these values $n_{S}^{ \pm}(T)$.

\section{Discussion}

Our numerical results indicate that the time evolution drives the system to a homogeneous state where all nodes belong either to $S$ - or $D$-state. The boundary between the basins of attraction is a surface in the threedimensional space of parameters: the clustering coefficient $\mathcal{C}$, the probability $p$ of the $D$-process, and the initial concentration of the $S$-nodes. The boundary consists of unstable fixed points. The data shown in Figure 5 indicate, that the transition between two homogeneous states is most sharp for $\mathcal{C}=0.5$, i.e. for the Watts-Strogatz network without rewiring. Once the rewiring introduces some local disorder, the movement of the boundary between the $S$-phase and the $D$-phase can be stuck on local configurations, and the related metastable states blur the transition.

The advantage of our method of evaluation of the activity of a contact process by counterbalancing it by another contact process is that we get a stationary state which is not a frozen absorbing state, but a result of a dynamic equilibrium. The "another process" plays a role of a scale, which allows to compare different processes; if a new process appears, its comparison with a given dynamics allows to evaluate its efficiency with respect to all processes which had been previously compared with it.

More generally, simulations of competing processes allow to evade the method of quasi-stationary states [3], which is commonly used when a finite system is trapped in an absorbing state (see Refs. [23-26] and references therein). Having two processes in the game, we can distinguish three possible outcomes: any of the processes wins or we have a dynamical equilibrium between them. With the third option in hand, the first two are not necessarily artifacts of the finiteness of the system. The sharp transition between two absorbing states, reported above for the Watts-Strogatz network, has an alternative in the form of the dynamical equilibrium, as observed in references $[11,12]$, where the rules of the $D$-process have been modified.

The work was partially supported by the Polish Ministry of Science and Higher Education and its grants for scientific research and by the PL-Grid Infrastructure.

Note added in proof. In reference [13], actually we use the invasion dynamics and not the voter dynamics, contrary to the terminology used there.

\section{References}

1. T.M. Liggett, Stochastic Interacting Systems: Contact, Voter and Exclusion Processes (Springer-Verlag, Berlin, 1999)

2. E.T. Harris, Ann. Probab. 2, 969 (1974)

3. J. Marro, R. Dickman, Nonequilibrium Phase Transitions in Lattice Models (Cambridge UP, Cambridge, 1999)

4. M.M. de Oliveira, R.V. Dos Santos, R. Dickman, Phys. Rev. E 86, 011121 (2012)

5. B. Oborny, G. Meszéna, G. Szabó, Oikos 109, 291 (2005)

6. F. Giargulo, S. Huet, Europhys. Lett. 91, 58004, (2010)

7. T.M. Liggett, Ann. Probab. 24, 1675, (1996)

8. M. Henkel, H. Hinrichsen, J. Phys. A 37, R117 (2004)

9. G. Ódor, R. Pastor-Satorras, Phys. Rev. E 86, 026117 (2012)

10. I. Jensen, Phys. Rev. Lett. 70, 1465 (1993)

11. M. Rybak, K. Kułakowski, Int. J. Mod. Phys. C 24, 1350012 (2013) 
12. M. Rybak, K. Kułakowski, Int. J. Mod. Phys. C 27, $1692002(2016)$

13. M. Rybak, K. Kułakowski, Acta Phys. Pol. A 44, 1007 (2013)

14. Competition and Coexistence, edited by U. Sommer, B. Worm (Springer-Verlag, Berlin, 2002)

15. B. Karrer, M.E.J. Newman, Phys. Rev. E 84, 036106 (2011)

16. D. O'Sullivan, G.L.W. Perry, Spatial Simulations: Exploring Pattern and Process (John Wiley \& Sons, Chichester, 2012)

17. D.J. Watts, S.H. Strogatz, Nature 393, 440 (1998)

18. B. Bollobás, Random Graphs, 2nd edn. (Cambridge UP, Cambridge, 2001)

19. C. Castellano, AIP Conf. Proc. 779, 114 (2005)

20. V. Sood, T. Antal, S. Redner, Phys. Rev. E 77, 041121 (2008)

21. K. Sznajd-Weron, J. Sznajd, Int. J. Mod. Phys. C 11, 1157 (2000)
22. K. Sznajd-Weron, M. Tabiszewski, A.M. Timpanaro, Europhys. Lett. 96, 48002 (2011)

23. R.S. Ferreira, S.C. Ferreira, Eur. Phys. J. B 86, 462 (2013)

24. R. Dickman, M.M. de Oliveira, Physica A 357, 134 (2005)

25. S.C. Fereira, R.S. Fereira, R. Pastor-Satorras, Phys. Rev. E 83, 066113 (2011)

26. E.A. van Doorn, P.K. Pollett, Eur. J. Oper. Res. 230, 1 (2013)

27. W. de Nooy, A. Mrvar, V. Batagelj, Exploratory Social Network Analysis with Pajek, 2nd edn. (Cambridge UP, Cambridge, 2012)

Open Access This is an open access article distributed under the terms of the Creative Commons Attribution License (http://creativecommons.org/licenses/by/4.0), which permits unrestricted use, distribution, and reproduction in any medium, provided the original work is properly cited. 